\title{
Attenuation of half sulfur mustard gas-induced acute lung injury in rats
}

\author{
Shannon D. McClintock, ${ }^{1}$ Laszlo M. Hoesel, ${ }^{1}$ Salil K. Das, ${ }^{2}$ Gerd O. Till, ${ }^{1}$ Thomas Neff, \\ Robin G. Kunkel, ${ }^{1}$ Milton G. Smith ${ }^{1}$ and Peter A. Ward ${ }^{1, *}$ \\ ${ }^{1}$ The University of Michigan Medical School, Department of Pathology, Ann Arbor, MI 48109, USA \\ ${ }^{2}$ Meharry Medical School, Department of Biochemistry, Nashville, TN, USA \\ 3 Amaox, Ltd, Paw Paw, MI 49079, USA \\ Received 12 January 2005; Revised 29 June 2005; Accepted 9 August 2005
}

\begin{abstract}
Airway instillation into rats of 2-chloroethyl ethyl sulfide (CEES), the half molecule of sulfur mustard compound, results in acute lung injury, as measured by the leak of plasma albumin into the lung. Morphologically, early changes in the lung include alveolar hemorrhage and fibrin deposition and the influx of neutrophils. Following lung contact with CEES, progressive accumulation of collagen occurred in the lung, followed by parenchymal collapse. The coinstillation with CEES of liposomes containing pegylated (PEG)-catalase (CAT), PEG-superoxide dismutase (SOD), or the combination, greatly attenuated the development of lung injury. Likewise, the co-instillation of liposomes containing the reducing agents, N-acetylcysteine (NAC), glutathione (GSH), or resveratrol (RES), significantly reduced acute lung injury. The combination of complement depletion and airway instillation of liposomes containing anti-oxidant compounds maximally attenuated CEES-induced lung injury by nearly $80 \%$. Delayed airway instillation of anti-oxidant-containing liposomes (containing NAC or GSH, or the combination) significantly diminished lung injury even when instillation was delayed as long as $1 \mathrm{~h}$ after lung exposure to CEES. These data indicate that CEES-induced injury of rat lungs can be substantially diminished by the presence of reducing agents or anti-oxidant enzymes delivered via liposomes. Copyright (c) 2005 John Wiley \& Sons, Ltd.
\end{abstract}

KEY WORDS: CEES; anti-oxidant liposomes; neutrophils; fibrosis; macrophages

\section{Introduction}

As is well known, mustard gas [bis (2-chloroethyl ethyl) sulfide], also known as sulfur mustard (HD), has long been known to be a vesicant in humans and, when inhaled, causes extreme lung damaging reactions (Eisenmenger et al., 1991; Khateri et al., 2003; Lakshmana Rao et al., 1999). In human survivors, progressive lung dysfunction due to pulmonary fibrosis is well documented (Emad and Rezaian, 1999). Not unexpectedly, HD is radiomimetic, teratogenic and mutagenic (Angelov et al., 1996; Dube et al., 1998). Currently, there is no effective therapy for either the vesicant-inducing properties of HD or for the outcomes that can lead to acute and progressive lung injury and death.

2-Chloroethyl ethyl sulfide (CEES) is less toxic than HD and can be used in the absence of facilities required for HD studies. In rats CEES has been shown

* Correspondence to: Dr Peter A. Ward, The University of Michigan Medical School, Department of Pathology, 1301 Catherine Rd, Ann Arbor, MI 48109, USA.

E-mail: pward@umich.edu

Contract/grant sponsor: USAMRMC DAMD; Contract/grant number: 17-032-0054. to induce acute lung injury in a dose-dependent and time-dependent manner (McClintock et al., 2002). CEESinduced acute lung injury is complement- and neutrophildependent, suggesting that some of the CEES-induced injury is due to engagement of the inflammatory response in lung in an unknown manner (McClintock et al., 2002). Furthermore, lung injury is attenuated after intravenous treatment with the anti-oxidant, $\mathrm{N}$-acetylcysteine (NAC), or airway delivery of anti-oxidants or anti-oxidant enzymes (McClintock et al., 2002). These data have suggested that CEES compromises the redox potential in the lung, putting it at risk of oxidant-mediated injury.

Liposomal delivery of drugs or chemical compounds is a way to achieve high tissue levels of a desired compound (Fan et al., 2000; Freeman et al., 1985; Suntres and Shek, 1996). In the lung, airway delivery of liposomes results in macrophage uptake of liposomes by a phagocytic pathway (Gonzalez-Rothi et al., 1991; Shephard et al., 1981; Sone et al., 1980). As far as is known, liposomes are not internalized by any other lung cells. The current studies demonstrated that liposomes containing anti-oxidants or anti-oxidant enzymes cause a reduction in acute lung injury in rats following airway delivery of CEES. Furthermore, delivery of such liposomes, when delayed $1 \mathrm{~h}$ after CEES administration, still provides significant attenuation of acute lung injury. 
These findings may have important therapeutic implications for HD-induced acute lung injury in humans.

\section{Materials and Methods}

\section{Chemicals}

Except where noted, all chemicals and reagents were purchased from the Sigma Chemical Co. (St Louis, MO).

\section{Animal Model}

Adult male (275-325 g) specific pathogen-free LongEvans rats (Harlan Co., Indianapolis, IN) were used in these studies. Intraperitoneal ketamine $\left(100 \mathrm{mg} \mathrm{kg}^{-1}\right.$ body weight) (Fort Dodge Animal Health, Fort Dodge, IA) was used for anesthesia and intraperitoneal xylazine (13 $\mathrm{mg} \mathrm{kg}^{-1}$ body weight) (Bayer Corp. Shawnee Mission, KS) was used for sedation when required (delayed time point liposome administration). The experimental procedure for CEES-induced lung injury in rats has been described previously (McClintock et al., 2002). Briefly, after induction of anesthesia, ${ }^{125}$ I-labeled bovine serum albumin ( ${ }^{125} \mathrm{I}-\mathrm{BSA}, 0.5 \mu \mathrm{Ci}$ per rat) was injected intravenously as a quantitative marker for vascular leakage. The trachea was then surgically exposed and a slightly curved P50 catheter was inserted into the trachea past the bifurcation so as to facilitate a unilateral, left-lung injury. A small volume of CEES ( $2 \mu \mathrm{l}$ per rat; about $\left.6 \mathrm{mg} \mathrm{kg}^{-1}\right)$ was solubilized in ethanol ( $58 \mu \mathrm{l}$ per rat) and then added to a syringe containing Dulbecco's phosphate buffered saline (DPBS) (340 $\mu \mathrm{l}$ per rat). This solution was injected via the intratracheal catheter, into the left lung main stem bronchus. Studies, not requiring the usage of a radiolabeled marker, proceeded identically substituting DPBS for the radioactive injection. For all studies, except the time response experiment, animals were killed $4 \mathrm{~h}$ later, the pulmonary arterial circulation was flushed with $10 \mathrm{ml}$ of cold DPBS, the lungs were surgically dissected, placed in counting vessels, and the amount of radioactivity ( ${ }^{125}$ I-labeled BSA) determined by gamma counting. For calculations of the permeability index, the amount of radioactivity ( ${ }^{125}$ I-labeled BSA) remaining in the lungs in which the vasculature was perfused with saline was divided by the amount of radioactivity present in $1.0 \mathrm{ml}$ of blood obtained from the inferior vena cava at the time of killing as described elsewhere (McClintock et al., 2002). ${ }^{125}$ I-BSA present in the lung after thorough flushing of the vasculature is a quantitative measure of the degree of vascular endothelial and alveolar epithelial damage, in which much of the ${ }^{125} \mathrm{I}-\mathrm{BSA}$ can be lavaged from the distal airway compartment, indicating the loss of the vascular and epithelial barriers (Johnson and Ward, 1974).
All animal experiments were in accordance with the standards in The Guide for the Care and Use of Laboratory Animals, and were supervised by veterinarians from the Unit for Laboratory and Animal Care of the University of Michigan Medical School.

\section{Complement Depletion}

Cobra venom factor (CVF) was purified from crude, lyophilized cobra venom (Naja atra) by ion exchange chromatography (Ballow and Cochrane, 1969). Complement depletion of experimental animals was achieved by intraperitoneal injections of 25 units of CVF per rat at time zero and $24 \mathrm{~h}$ later, resulting in undetectable levels of serum hemolytic complement activity as confirmed by CH50 assay (Mayer, 1961). Experiments were performed $24 \mathrm{~h}$ after the second CVF injection.

\section{Liposome Preparation}

Dipalmitoylphosphatidylcholine (DPPC, Avanti Polar Lipids) was dissolved $20 \mathrm{mg} \mathrm{ml}^{-1}$ in a $2: 1 \mathrm{v} / \mathrm{v}$ chloroform/methanol solution. When $\alpha$-tocopherol $(\alpha \mathrm{T})$ was also included in the liposomes, it was added just after the chloroform/methanol solvent to provide a $7: 3$ molar ratio (DPCC : $\alpha$-T) after first being carefully dissolved in a small volume of ethanol. The DPPC or (DPPC : $\alpha$-T) solution was then dried under a thin stream of nitrogen in a round bottom flask to form a thin lipid film on the walls of the tube. Once the film had been dried, the tube was then placed on a vacuum for at least $1 \mathrm{~h}$ to further dry and remove any excess organic compounds from the lipid film.

The compounds being encapsulated in the liposomes were exclusively prepared in Dulbecco's phosphate buffered saline (DPBS), $\mathrm{pH}$ adjusted to 7.4 and then added to the lipid film. The tube was then vortexed to free the lipid film from the walls of the tube, and then placed in a heated water bath $\left(41^{\circ} \mathrm{C}\right)$. When sizing the liposomes, it is necessary to keep them at a temperature above their transition phase. The transition phase temperature for DPPC is $41^{\circ} \mathrm{C}$. Vortexing the liposomes once they are above the transition phase temperature results in large multilamellar vesicles. To reduce the size of the vesicles and to produce uniform small unilamellar vesicles, the lipid suspension was then passed ten times through polycarbonate membrane filters in a Liposofast Basic mini extruder available from Avestin, Inc. (Ottawa, Ontario). The resulting liposomes were uniform in size measuring $100 \mathrm{~nm}$ in diameter. According to the manufacturer, the use of an extruder is an efficient method for producing liposomes that are of relatively uniform size. Liposomes were checked via light microscopy for uniformity and size. Liposomes were injected intratracheally in a volume 
of $100 \mu \mathrm{l}$ per rat through the same catheter setup used for CEES instillation at the time point designated by each individual experimental protocol.

\section{Morphological Assessment of Lung Injury}

In order morphologically to assess lung injury, lungs were fixed by intratracheal instillation of $10 \mathrm{ml}$ buffered $(\mathrm{pH}$ 7.2) formalin $(10 \%)$ at the indicated time points following airway instillation of CEES. Lung sections were then obtained for histological examination by staining with hematoxylin and eosin. In addition, lung sections were stained with trichrome in order to assess the deposition of fibrin and collagen (Luna, 1968).

\section{Statistical Analysis}

The results are presented as mean \pm SEM in the text and figures. Groups $(n \geq 5)$ were subjected to oneway analysis of variance and when significance was found, Student's $t$-test with the Bonferroni correction for multiple comparisons was applied. A value of $P<0.05$ was considered significant.

\section{Results}

\section{Histopathologic Features of Lung Response to CEES}

Following airway instillation of CEES into rat lungs, tissues were obtained at $0,6,12$ and $24 \mathrm{~h}$ as well 3 and 6 days and 6 weeks after exposure to CEES. Lung sections were stained with trichrome stain to evaluate lung deposition of fibrin and collagen. Composite results are shown in Fig. 1 (frames A-I). At time 0, trichrome stains revealed the usual perivascular and septal evidence of collagen (frames A, B). As early as $6 \mathrm{~h}$, increased evidence of trichrome stained deposition in alveolar walls was likely related to fibrin deposition (frame C). By $24 \mathrm{~h}$ after lung instillation of CEES, dense interstitial and intra-alveolar accumulations of trichrome positive (blue dye) were evident throughout the affected lungs, suggestive of increased deposition of fibrin and collagen fibers (frame D). Intra-alveolar hemorrhage, edema and intraalveolar accumulation of macrophages and mononuclear cells were found at $24 \mathrm{~h}$ (frame E). By 3 days, dense interstitial deposits of fibrin and collagen occurred (frame F). By day 6, extensive confluent collagen deposits were found in the lung, together with a collapse of alveolar

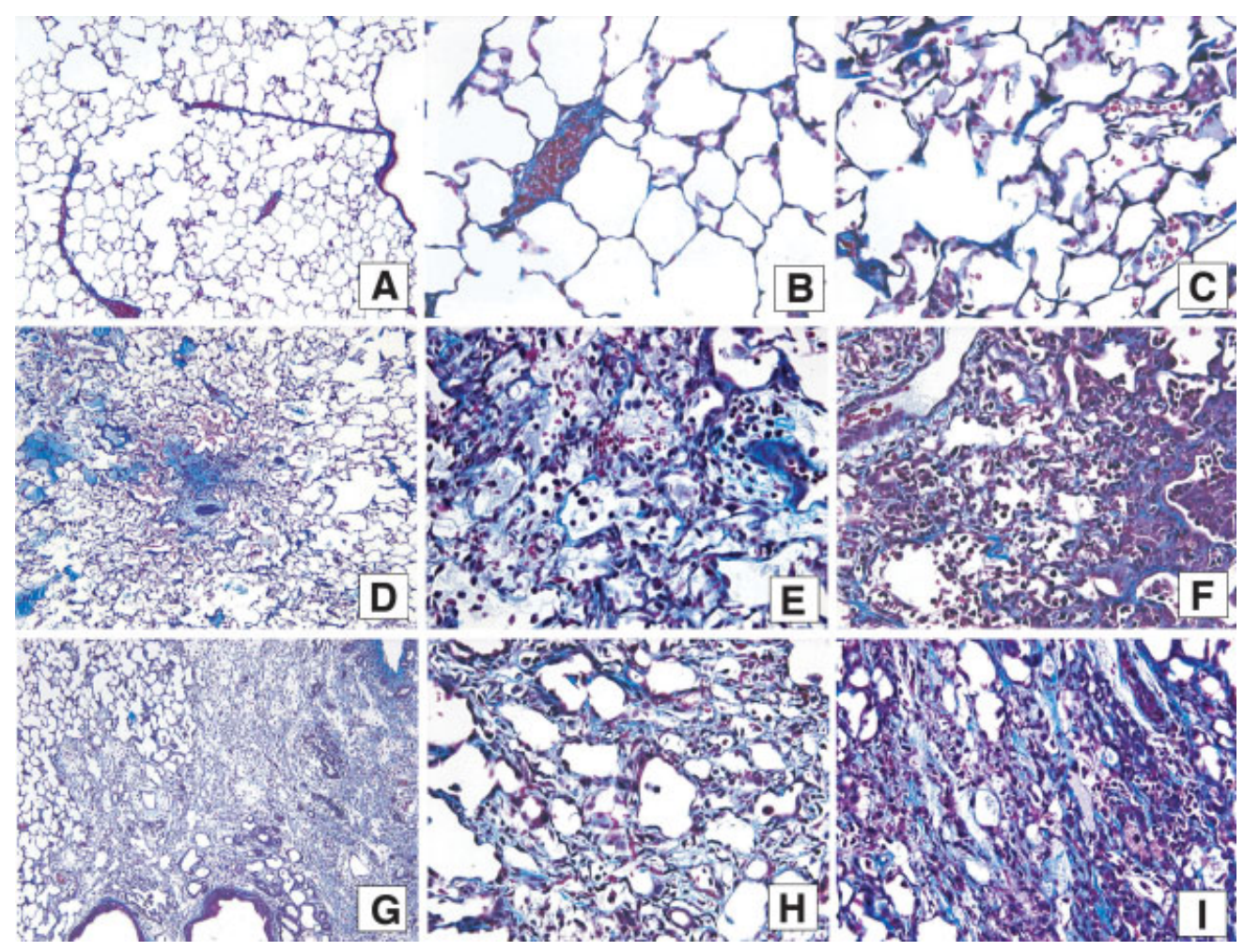

Figure 1. Tissue sections of lungs with trichrome stain. Lungs were obtained after airway instillation of CEES at time 0 ( $A, B, 10 \times$ and $40 \times) ; 6 h(C, 40 x) ; 24 h(D, E, 10 \times$ and 40x); 3 days $(F, 10 \times), 6$ days $(G, 10 \times$ and $H, 40 x)$; and 3 weeks $(I, 40 \times)$. All tissue sections were reacted with trichrome stain 


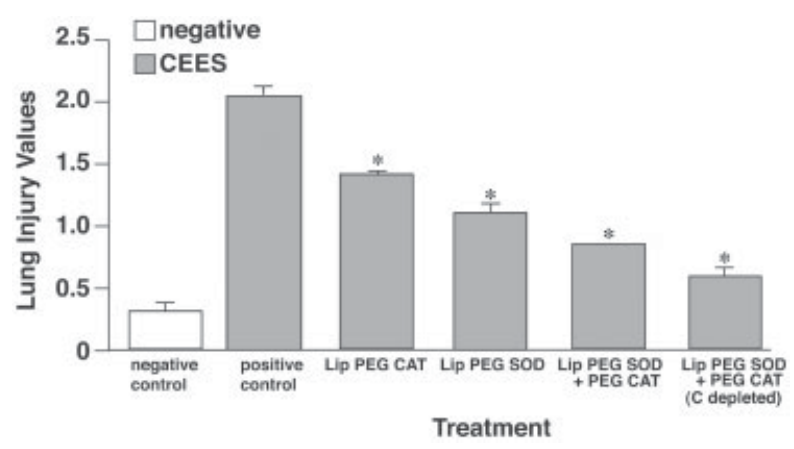

Figure 2. Attenuative effects of liposomes loaded with anti-oxidant enzymes. Rats received either saline followed by unloaded liposomes (negative control), CEES followed by airway delivery of unloaded liposomes (positive control), or CEES with liposomes containing either pegylated (PEG)-CAT or PEG-SOD, individually or the combination, or the combination of liposomes in complement depleted animals induced by the earlier intraperitoneal injection of purified cobra venom factor. Liposomes were administered immediately after CEES instillation. For each bar $n \geq 6$. Lung injury values are represented by the leak of ${ }^{125}$ albumin from the vascular compartment into the airway compartment $4 \mathrm{~h}$ after airway delivery of CEES (see text). * Represents $P$ values of $<0.05$ when compared with the positive control group

structures and the appearance of honeycombing (frames G, H). By week 3, little recognizable lung structure remained in the face of dense collagen deposits and parenchymal collapse, together with numerous interstitial macrophages and mononuclear cells (frame I).

\section{Attenuation of CEES-Induced Acute Lung Injury by Anti-Oxidant Enzymes in Liposomes}

As shown in Fig. 2, the airway instillation of CEES together with unloaded liposomes resulted $4 \mathrm{~h}$ later in approximately a 10-fold increase in lung injury, as defined by the leakage of ${ }^{125} \mathrm{I}$-albumin from blood into the lung. When instilled into the lung immediately after CEES, polyethyleneglycol (PEG)-linked catalasecontaining liposomes (LIP-PEG-CAT) attenuated injury by $40 \%$. Liposomes containing PEG-superoxide dismutase (PEG-SOD) diminished injury by $57 \%$. The combination of PEG-SOD and PEG-CAT in liposomes further reduced injury by $71 \%$. With the combination of PEG-SOD and PEG-CAT liposomes given to complement-depleted animals, the injury was reduced by $86 \%$. These data indicate that anti-oxidant enzymes have powerful attenuative effects on CEES-induced acute lung injury. Since, as described above, airway delivery of liposomes results in their phagocytosis by lung macrophages, it seems likely that the attenuative effects of liposomes

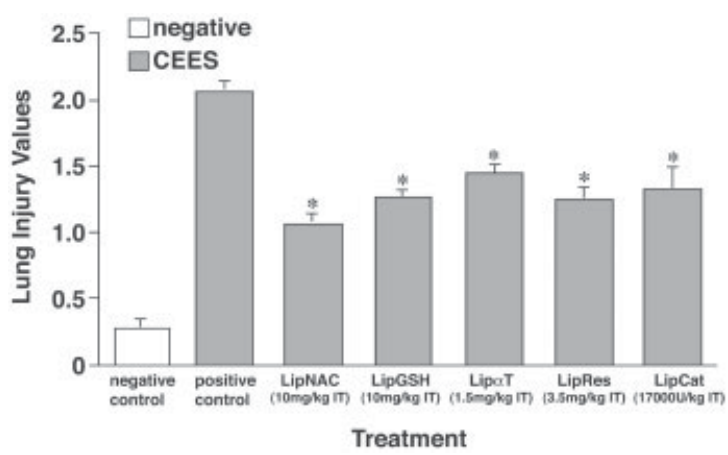

Figure 3. Attenuative effects of liposomes loaded with reducing agents in CEES lung injury. The positive and negative controls are similar to those described in Fig. 1. When used, liposomes were injected intratracheally immediately after the airway instillation of CEES. Lung injury was determined by the permeability index. $\alpha \mathrm{T}, \alpha$-tocopherol

containing anti-oxidant enzymes are due to the bolstering of anti-oxidant defenses in lung macrophages.

\section{Attenuative Effects of Liposomes Containing Reducing Agents}

In an additional set of experiments (shown in Fig. 3), there was approximately a 10-fold increase in leakage of albumin from the circulation into the lungs of animals receiving airway instillation of CEES $4 \mathrm{~h}$ earlier together with unloaded liposomes. When liposomes containing NAC (Lip-NAC) were instilled immediately after CEES, injury was attenuated by $60 \%$. Liposomes containing glutathione (GSH) led to a $48 \%$ reduction in lung injury. Liposomes containing $\alpha$-tocopherol $(\alpha \mathrm{T})$ reduced injury by $37 \%$. Liposomes containing the reducing agent present in red wine, resveratrol (RES), reduced injury by $48 \%$, while liposomes containing PEG-CAT reduced injury by $44 \%$. These data indicate that reducing agents presented in liposomes have significantly attenuative effects against CEES-induced acute lung injury. The data also indicate that the non-derivatized form of catalase (CAT) also has attenuative effects when given within liposomes.

\section{Additive Effects of Complement Depletion and Liposomes Containing Reducing Agents}

Previous studies in our laboratory have shown that complement depletion resulted in a $43 \%$ reduction of lung injury (McClintock et al., 2002). As shown in Fig. 4, approximately a 10-fold increase in the leakage of ${ }^{125}$ I-albumin into the lungs occurred following instillation of CEES together with unloaded liposomes. When the animals were complement (C) depleted, the instillation 


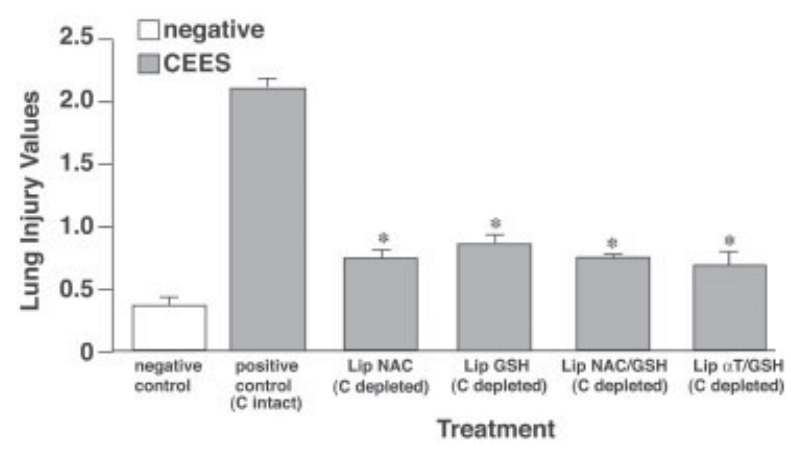

Figure 4. Enhanced effects of anti-oxidant containing liposomes in complement depleted rats. The negative and positive control groups are similar to those described in Fig. 1. Complement depletion was induced in four groups of animals by the prior intraperitoneal injection of purified cobra venom factor (CVF). Liposomes containing the various anti-oxidant compounds were given immediately after airway instillation of CEES. For each group, $n \geq 6$

of liposomes containing NAC reduced injury by $79 \%$, those containing GSH reduced injury by $72 \%$ in complement-depleted rats, liposomes containing the combination of NAC and GSH reduced injury by $78 \%$ in complement-depleted rats. Complement-depleted animals receiving liposomes containing $\alpha \mathrm{T}$ together with GSH showed an $82 \%$ reduction in lung injury as measured by leakage of albumin from the blood. Thus, the combination of complement depletion and anti-oxidant liposomes seems significantly to attenuate CEES-induced acute lung injury in an additive manner.

\section{Effects of Delayed Lung Instillation of Anti-Oxidant Liposomes}

As shown in Fig. 5, in CEES treated animals instillation of liposomes containing reducing agents was done either $10 \mathrm{~min}$ before the airway instillation of CEES or at 30, 60 and, in one case, 90 min following the airway instillation of CEES. As shown in Fig. 5, over the course of the first $60 \mathrm{~min}$ after instillation of CEES, there were significant attenuative effects of liposomes containing NAC or GSH, or the combination. Under these circumstances, injury was reduced between 55\% and 77\%, respectively. In the case of liposomes containing the combination of NAC and GSH, even when delivery was delayed until 90 min following instillation of CEES, there was a $55 \%$ reduction in the development of acute lung injury. These data indicate that delayed airway administration of anti-oxidant-containing liposomes results in significant reduction of CEES-induced lung injury, even when delivery is delayed by at least an hour following exposure of lungs to CEES.

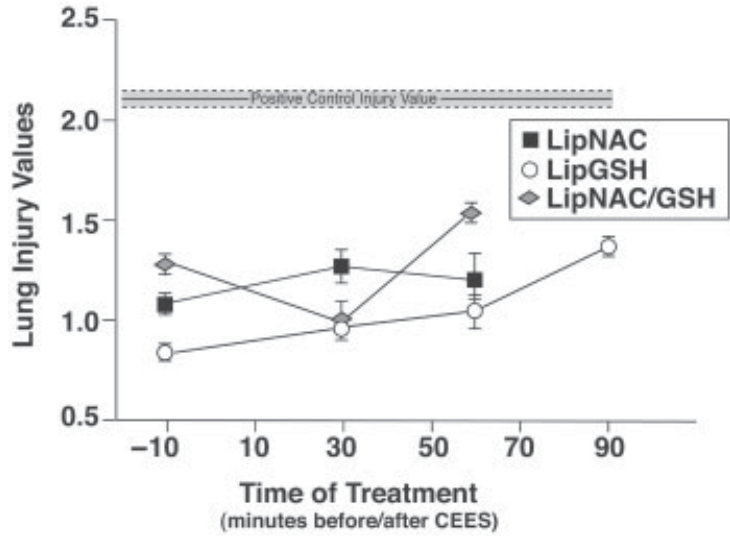

Figure 5. Attenuative effects of anti-oxidant containing liposomes when airway instillation was given at various time points before or after airway delivery of CEES. The dotted line near the top represents the positive control value. Lung injury values (permeability indices) in the negative control are not shown but were $<0.5$, as described in Figs $1-3$

\section{Discussion}

The data described in this report indicate that CEES instillation into the lung produces acute lung injury in a manner that seems related to the loss of the redox balance in the lung, although this has not been demonstrated directly. This conclusion is based on the attenuative effects of reducing agents (NAC, GSH, $\alpha \mathrm{T}$, resveratrol) or anti-oxidant enzymes (SOD, CAT) or various combinations, all presented in liposomes alone or in combination. Since it is well known that liposomes given into the airways are phagocytized by macrophages and internalized (Gonzalez-Rothi et al., 1991; Lentsch et al., 1999; Shephard et al., 1981), the implications from the current studies are that liposomal delivery selectively enhances a reducing environment in lung macrophages, which may be compromised when these cells came into contact with CEES.

The morphological features described in this report are consistent with our earlier report of an accumulation of myeloperoxidase (MPO) in the lung after CEES instillation (McClintock et al., 2002). The presence of alveolar hemorrhage and edema implies a severe disruption of vascular and distal airway barrier. These changes are consistent with the concept that CEES induces an acute lung-damaging inflammatory response that is complement-dependent. Beneficial effects of neutrophil and complement depletion as demonstrated previously indicate that the inflammatory response to CEES contributes to the development of lung injury (McClintock et al., 2002). Masson's trichrome staining revealed an accumulation of fibrin and/or collagen within the alveolar spaces. Deposition of fibrin reflects a nonspecific reaction to tissue damage. It remains to be shown 
that CEES-exposure causes rapid development of interstitial fibrosis, as confirmed biochemically by collagen accumulation. It is hypothesized that epithelial and endothelial damage following CEES-exposure results in disruption of tissues, resulting in collagen accumulation in the interstitial and alveolar spaces. It appears likely that following lung exposure to CEES collagen deposition occurs in a widespread manner, resulting in parenchymal collapse and the honeycombing changes that occur in humans with pulmonary fibrosis. Numerous macrophages and mononuclear cells in areas of collagen deposition in the lung may be associated with the release of mediators (such as $\mathrm{TGF}_{\beta}$ ) that promote lung production of collagen.

How CEES functions as a powerful oxidant and what lung cells are targets of CEES is unclear. The extensive leakage of albumin into the lung after exposure to airway administration of CEES infers that the blood-gas barrier has been seriously compromised, causing a functional impairment (or destruction) of both vascular endothelial and alveolar epithelial cells. The subsequent alveolar flooding with plasma components leaking into the distal airway compartment could seriously compromise bloodgas exchange, resulting in hypoxia.

The permeability index after instillation of CEES and empty liposomes was found to be similar to CEES instillation alone (as reported in McClintock et al., 2002) implying that empty liposomes do not cause any lung damage by themselves. The fact that the combination of complement depletion and liposomal delivery of GSH or NAC enhances the attenuation when compared with the use of either type of liposomes given to complementintact rats (Figs 3 and 4) suggests that it may be both the loss of reducing potential in the lung as well as an engagement of complement activation products (e.g. C5a) that leads to intense acute lung injury. Whether complement activation products are directly responsible for lung injury or are functioning to enhance cytokine and chemokine expression remains to be determined. In a recent study it was shown that neutrophil depletion prior to CEES delivery was also capable of reducing CEESinduced lung injury (McClintock et al., 2002), suggesting that activated neutrophils enter into the sequence of destructive events after CEES instillation into the lung.

A matter of considerable interest is that delayed delivery (for as long as $60 \mathrm{~min}$ ) of liposomes containing NAC or GSH, or the combination, into lungs after CEES instillation still provides substantial attenuation from the massive leak of albumin into the lung. It should be noted that there is not much increased albumin leak into the lung in the first $60 \mathrm{~min}$ after administration of CEES (McClintock et al., 2002). In fact, compared with values at $1 \mathrm{~h}$, the permeability index at 2, 4 and $6 \mathrm{~h}$ after instillation of CEES rose 2 fold, 2.9 fold, 7.7 fold and 16.2 fold, respectively, when compared with uninjured lung values. Accordingly, the development of extensive lung injury after airway instillation of CEES requires considerable time for full development of lung injury. This would be in accord with the concept that CEES triggers in the lung an acute inflammatory response, which itself serves to cause lung damage. Since this sequence requires several hours before the large increases in lung permeability (albumin leak into lung) are seen, this may explain why delayed administration of anti-oxidant liposomes can still bring about significant attenuative effects. Understanding more fully the molecular events that lead to CEES-induced intense acute lung injury may provide even better strategies for effective therapeutic intervention after exposure of lung to HD and related compounds.

\section{References}

Angelov A, Belchen L, Angelov G. 1996. Experimental sulfur mustard gas poisoning and protective effect of different medicines in rats and rabbits. Indian Vet. J. 73: 546-551.

Ballow M, Cochrane CG. 1969. Two anticomplementary factors in cobra venom: hemolysis of guinea pig erythrocytes by one of them. J. Immunol. 103: 944-952.

Dube SN, Husain K, Sugendran K, Vijayaraghavan R, Somani SM. 1998. Dose response of sulphur mustard: behavioral and toxic signs in rats. Indian J. Physiol. Pharmacol. 42: 389-394.

Eisenmenger W, Drasch G, von Clarmann M, Kretschmer E, Roider G. 1991. Clinical and morphological findings on mustard gas [bis(2chloroethyl)sulfide] poisoning. J. Forensic Sci. 36: 1688-1698.

Emad A, Rezaian GR. 1999. Immunoglobulins and cellular constituents of the BAL fluid of patients with sulfur mustard gas-induced pulmonary fibrosis. Chest 115: 1346-1351.

Fan J, Shek PN, Suntres ZE, Li YH, Oreopoulos GD, Rotstein OD. 2000. Liposomal antioxidants provide prolonged protection against acute respiratory distress syndrome. Surgery 128: 332-338.

Freeman BA, Turrens JF, Mirza Z, Crapo JD, Young SL. 1985. Modulation of oxidant lung injury by using liposome-entrapped superoxide dismutase and catalase. Fed. Proc. 44: 2591-2595.

Gonzalez-Rothi RJ, Straub L, Cacace JL, Schreier H. 1991. Liposomes and pulmonary alveolar macrophages: functional and morphologic interactions. Exp. Lung Res. 17: 687-705.

Johnson KJ, Ward PA. 1974. Acute immunologic pulmonary alveolitis. J. Clin. Invest. 54: 349-357.

Khateri S, Ghanei M, Keshavarz S, Soroush M, Haines D. 2003. Incidence of lung, eye, and skin lesions as late complications in 34,000 Iranians with wartime exposure to mustard agent. J. Occup. Environ. Med. 45: 1136-1143.

Lakshmana Rao PV, Vijayaraghavan R, Bhaskar AS. 1999. Sulphur mustard induced DNA damage in mice after dermal and inhalation exposure. Toxicology 139: 39-51.

Lentsch AB, Czermak BJ, Bless NM, Van Rooijen N, Ward PA. 1999. Essential role of alveolar macrophages in intrapulmonary activation of NF-kappaB. Am. J. Respir. Cell Mol. Biol. 20: 692-698.

Luna L. 1968. Manual of Histologic Staining Methods of the AFIP, 3rd edn. McGraw-Hill: New York.

Mayer MM. 1961. Complement and complement fixation. In Experimental Immunochemistry, Kabat EA, Mayer MM (eds). Springfield: Thomas, 133-240.

McClintock SD, Till GO, Smith MG, Ward PA. 2002. Protection from half-mustard-gas-induced acute lung injury in the rat. J. Appl. Toxicol. 22: 257-262.

Shephard EG, Joubert JR, Finkelstein MC, Kuhn SH. 1981. Phagocytosis of liposomes by human alveolar macrophages. Life Sci. 29: 2691-2698.

Sone S, Poste G, Fidler IJ. 1980. Rat alveolar macrophages are susceptible to activation by free and liposome-encapsulated lymphokines. J. Immunol. 124: 2197-2202.

Suntres ZE, Shek PN. 1996. Treatment of LPS-induced tissue injury: role of liposomal antioxidants. Shock 6 (Suppl 1): S57-S64. 